\title{
La representación social como categoría teórica y estrategia metodológica
}

\author{
The social representation as a theoretical category and \\ methodological strategy
}

\author{
Clara Betty Weisz ${ }^{1}$ ORCID \\ 'Universidad de la República \\ Uruguay
}

Fecha correspondencia:

Recibido: abril 11 de 2016.

Aceptado: febrero 6 de 2017.

Forma de citar:

Weisz, C.B., (2017). ). La

representación social como

categoría teórica y estrategia

metodológica. Rev. CES Psicol.,

10(1), 99-108.

Open access

(c) Copyright

Licencia creative commons

Etica de publicaciones

Revisión por pares

Gestión por Open Journal System

DOI: http://dx.doi.org/10.21615/

cesp.10.1.7

ISSN: 2011-3080

Sobre los autores:

1. Magíster y Licenciada en

Sociología. Docente del Instituto de Fundamentos y Métodos de la

Psicología, Programa Fundamentos Interdisciplinarios de la Psicología en la Hipermodernidad, facultad de Psicología, Universidad de La República, Uruguay.

Comparte

70 G. $898 \mathbb{R}^{\circ}$ 四

\section{Resumen}

El presente artículo lleva a cabo una revisión temática de la noción de Representación Social, en tanto categoría teórica y herramienta metodológica, en el entendido que su potencialidad heurístico, posibilita el estudio en profundidad de los sentidos y significantes que los sujetos le otorgan a la acción en la vida cotidiana; objetivo central de la metodología cualitativa de investigación en Ciencias Sociales. La vigencia y relevancia de dicha categoría conduce a la articulación de las diferentes tradiciones teóricas que le dieron origen, al abordaje de las técnicas relativas a la construcción del dato y al desarrollo de los modos que adoptan respectivamente, la interpretación y el análisis, hacia la producción de conocimiento contextualizado.

Palabras clave: Representación Social, Teoría Social, Investigación Cualitativa, Metodología.

\section{Abstract}

This article conducts a thematic revision of the social representation concept, both in theoretical category and methodological tool, implying that its heuristic potential allows studying in-depth the significance and sense, individuals have devoted to the action during daily life, which is the main purpose of the qualitative research methodology in Social Sciences. The validity and relevance of this category lead to articulate the different traditional theories by approaching the techniques related to the data construction and the development types that adopt the interpretation and analysis towards the Production of contextualized knowledge.

Keywords: Social Representation, Social Theory, Qualitative Research, Methodology.

\section{Introducción}

El presente artículo se centra en la categoría teórica de Representación Social (RS) como concepto central para la construcción de conocimiento, desde una postura epistemológica que plantea el regreso del sujeto (Ibáñez, 1994), evitando la trampa de una supuesta objetividad y externalidad del objeto de estudio, ya que en Ciencias Sociales no se trata de una rela- 
Las experiencias vividas, la transmisión intergeneracional y los modos de comunicación van configurando representaciones fragmentadas en función de los desiguales espacios que habitamos en las sociedades hipermodernas hoy (Weisz, 2014). La construcción de sentido, de subjetividad y las identidades móviles fluctúan en el marco de las actuales sociedades de riesgo, generando incertidumbre, fragilización de los vínculos y lazos sociales, obstaculizando la consolidación de sujetos colectivos. ción sujeto-objeto sino que todo proceso de investigación conlleva un vínculo dialógico sujeto-sujeto.

La RS, en tanto sistema socio cognoscitivo de sentidos y significantes culturalmente construidos y reproducidos por el sujeto en todo vínculo e interacción, constituye en sí misma el dato principal. Este en tanto, no puede ser considerado como un objeto a ser recolectado, sino que es producido en el proceso de co-construcción entre el investigador y los sujetos participantes de la investigación, principalmente a partir de dispositivos grupales.

La RS se presenta entonces, particularmente pertinente para llevar adelante todos aquellos estudios sociales, culturales y psicosimbólicos, ya que tiene la capacidad de potenciar y posibilitar el despliegue del sentido común y de la subjetividad, característica específica de la metodología cualitativa, que busca comprender e interpretar los sentidos y significados que los actores le otorgan a los fenómenos de los cuales cotidianamente son parte, y por tanto, actúan en consecuencia.

Hacer explícito y manifiesto el sentido común consensuado y hegemónico sobre determinada representación social -ya sea que se trate de juventud, infancia, consumo problemático, el lugar simbólico de los integrantes del núcleo familiar, el sentido del trabajo, o la credibilidad y legitimidad el sistema político, entre otros-, es una tarea compleja en el marco de la fragmentación y polarización de las actuales sociedades líquidas (Bauman, 2000, 2005) e hipermodernas (Aubert, 2004; Lipovetsky, 2006; Araújo, 2013). Llegar a una única concepción consensuada era más viable en el marco de las certezas constitutivas de la modernidad.

Las experiencias vividas, la transmisión intergeneracional y los modos de comunicación van configurando representaciones fragmentadas en función de los desiguales espacios que habitamos en las sociedades hipermodernas hoy (Weisz, 2014). La construcción de sentido, de subjetividad y las identidades móviles fluctúan en el marco de las actuales sociedades de riesgo, generando incertidumbre, fragilización de los vínculos y lazos sociales, obstaculizando la consolidación de sujetos colectivos. En este sentido los diversos fragmentos y estratos socioeconómicos y culturales, así como las diferencias generacionales van conformando diferentes, yuxtapuestas y hasta contrapuestas representaciones sociales. Visibilizar y desnaturalizar las representaciones que están presentes y subyacen en los diversos ámbitos y sectores poblacionales, genera necesariamente reflexividad sobre el mundo de la vida y la cotidianeidad. Y toda reflexividad abre las condiciones de posibilidad de transformación y de cambio.

En este sentido el presente artículo, retoma la noción de RS en tanto categoría teórica, articulando las dos principales perspectivas en que se fundamenta. Por un lado, la propuesta de Moscovici (1986), quien basándose en la concepción durkhemiana, privilegia el aspecto cognoscitivo y comunicacional; y por el otro, la tradición weberiana, que desde la fenomenología y del interaccionismo simbólico, concibe la RS como producto de la interacción intersubjetiva socialmente construida. Retomar ambas tradiciones no responde a una postura ecléctica, más bien, busca articularlas en el entendido que es posible y necesario, considerar aspectos de cada una de dichas tradiciones al momento de profundizar en la RS como categoría teórica.

Así mismo, se presenta la potencialidad de la RS como estrategia metodológica que habilita el conocimiento del imaginario colectivo — siguiendo a Durkheim-, o, desde la perspectiva de Weber, el sentido mentado de la acción (Weber, 1921/1985). 
Más allá de las diferencias y especificidades de cada una de dichas perspectivas, en ambas los abordajes grupales parecen ser los más pertinentes, en tanto el sujeto está siempre dentro de un sistema social y semiótico, es producto y productor de un saber acumulado y trasmitido intergeneracionalmente, a la vez que responde a un deber ser coercitivo, producto del proceso de socialización.

Lo antedicho no desconoce que la RS presenta siempre desafíos metodológicos, ya sea que se realicen Grupos de Discusión -más consistentes con la perspectiva durkhemiana de la coerción y del deber ser-; o que se realicen Grupos Focales más acordes a la perspectiva weberiana- en los que, desde el saber y el principio de realidad, se construyen las tipologías que dan sentido y orientan las acciones e interacciones intersubjetivamente mediadas (Canales, 2006).

En el presente artículo se describen por tanto, las características de cada una de las técnicas de construcción del dato mencionadas, analizando sus alcances y límites. Y siguiendo la lógica del diseño de investigación, se aborda las diferentes estrategias utilizadas en la etapa del análisis de lo producido en dichas instancias conversacionales.

Al interpretar lo producido grupalmente, también se pueden distinguir estrategias de análisis que derivan de la perspectiva que predomine. El Análisis del Discurso suele ser la herramienta de análisis elegida para describir los códigos que los sujetos despliegan desde los ideales sociales y la normatividad orientada por el principio de deber (Canales, 2006), derivada de la tradición durkhemiana. Mientras que la Teoría Fundamentada en los Datos o Grunded Theory (Huberman \& Miles, 1991), es particularmente pertinente para la consecución de la producción de conocimiento en torno a la RS emergente y subyacente en los discursos, más próxima a la verstehen weberiana, que enfatiza en el saber, el sentido común y el acontecer fenomenológico de la vida cotidiana.

En ambos casos, ya sea se lleve adelante un análisis del discurso del texto producido en la grupalidad o que se trabaje desde la codificación abierta y axial de la teoría fundamentada, se parte del supuesto de que el sustento de la RS se encuentra en la posibilidad de interpretación de los discursos, que no requieren verificación, demostración o contrastación externa.

En síntesis, comprender el vínculo entre la RS y la acción, la relación entre el modo en que se estructuran y organizan las visiones del mundo, y el modo en que se expresan en la vida cotidiana, constituye uno de los principales desafíos. En ese sentido el artículo desarrolla una revisión temática de la RS como categoría teórica, reconstruye la historia de su surgimiento y plantea las referencias teóricas en que se fundamenta; seguidamente desarrolla los aspectos relativos a la metodología, y por último aborda los desafíos vinculados al análisis. Por su parte, las consideraciones finales sintetizan lo desarrollado, a la vez que proponen desafíos y abren nuevas interrogantes teórico-metodológicas.

\section{La representación social como categoría teórica}

La RS, tal como se entiende actualmente, se distancia de la concepción original de la lógica aristotélica (Aristóteles, 1994) en la que las categorías solo agrupan u organizan taxonómicamente contenidos, sin afirmar ni negar nada; así como también se contrapone a la perspectiva kantiana (Kant, 1781/2007), en la que las categorías son entendidas como conceptos puros y a priori del entendimiento. 
Pág 102

Una Representación Social es el resultado de la relación entre un objeto (algo o alguien o un evento) de representación y de un sujeto que lo representa (individuos y grupos) dentro de un marco histórico y cultural de referencia. De esta manera, podemos decir que las Representaciones Sociales son una forma de pensamiento socialmente elaborado y con una finalidad práctica, que permite la socialización y la comunicación entre miembros de un mismo grupo e incluso, con miembros de grupos diferentes.
En tanto categoría analítica, el origen de la RS se encuentra en el pragmatismo fundado por Pierce (1903/2007), quien delimita cuatro formas a través de las cuales es posible producir conocimiento acerca de toda categoría: a) el modo inferencial por abstracción; b) la distribución por extensión; c) el análisis metódico d) el hallazgo, descripción y selección de una propiedad, a partir de la cual se generan los conceptos, que a su vez se articulan con la teoría general. Es este último abordaje el que da origen a la teoría fundada (Acosta, Quiñones, \& Supervielle, 2015), particularmente relevante ya que es el mayormente utilizado en el análisis de las RS, que se fundamenta en la tradición weberiana.

Desde las perspectivas contemporáneas (Arbic, 2001; Joldelet, 1986; Martinic, 2006), se entiende que toda RS es relacional, es decir, supone siempre la existencia de otras categorías, por lo que el sentido no se produce de forma aislada e individual sino que se construye en relación con otros sujetos y con otras categorías. Es por ello que toda RS toma elementos del trasfondo común, que denominamos Imaginario Social (Castoriadis, 1980), entendido como el universo de significaciones imaginarias que operan como organizadores, delimitando lo instituido como lo legítimo. Es a partir de estas formas creadas por cada sociedad, instituciones como la familia, el sistema educativo o el sistema político, que se instituye como conjunto de normas, valores, orientaciones y finalidades de la vida, tanto individuales como colectivas.

Es, en el marco del Imaginario Social (IS) que caracteriza y predomina en cada momento socio-histórico, desde la herencia greco-latina hasta la hipermodernidad actual, que se construyen las representaciones sociales. Éstas conllevan un menor nivel de generalización que el IS, en tanto toda RS es de alguien, sobre algo, mientras que el IS refiere a visiones del mundo, desde las cuales las RS cobran sentido.

La RS, se inscribe entre lo psíquico y lo social, permite el interjuego entre lo macro y lo micro, entre la acción y la estructura, lo individual y lo colectivo. La articulación entre las condiciones objetivas y la subjetividad remiten a un sujeto que, lejos de ser un individuo aislado, interioriza y se apropia de las representaciones, interviniendo al mismo tiempo en su construcción y reproducción (Jodelet, 2008).

Una Representación Social es el resultado de la relación entre un objeto (algo o alguien o un evento) de representación y de un sujeto que lo representa (individuos y grupos) dentro de un marco histórico y cultural de referencia. De esta manera, podemos decir que las Representaciones Sociales son una forma de pensamiento socialmente elaborado y con una finalidad práctica, que permite la socialización y la comunicación entre miembros de un mismo grupo e incluso, con miembros de grupos diferentes. Dicho de otro modo, las representaciones sociales definen y producen la particularidad de los grupos sociales (Navarro \& Restrepo, 2013, p. i).

\section{El abordaje metodológico del estudio de las representaciones sociales}

El desafío metodológico consiste en contar con herramientas y procedimientos que permitan dar cuenta de las tres dimensiones centrales constitutivas de las RS, o sea de todo sistema sociocultural de sentidos compartidos. La RS está formada por el contenido informacional; por cierto orden y coherencia interna que conforma categorías que constituyen la estructura de la representación; y por un orden ético-normativo por el cual las RS "son construcciones válidas para el sujeto y adquieren carácter de verdad legítima o de realidad deseable en base a sus creencias, formación y contexto cultural del cual forma parte" (Martinic, 2006, p. 300). 
De las dos perspectivas principales sobre las que se fundamenta la categoría RS, derivan diferentes estrategias metodológicas.

Por su parte, desde la perspectiva weberiana de la acción, el estudio de las RS, en tanto categoría hermenéutica, se focaliza en las interacciones simbólicamente mediadas, consideradas como "construcción social de la realidad", (Berger \& Luckmann, 1986) que suceden fenomenológicamante en toda interacción intersubjetiva. Por lo cual, desde esta perspectiva, la RS no queda anclada en el plano mental, sino que constituye la realidad e intersubjetividad desde el saber del sentido común, desde la vivencia de la vida cotidiana. La RS puede ser dicha, pensada o actuada, se manifiesta y expresa a través de palabras, gestos y acciones. Lo representacional entonces, tiene repercusiones en la práctica, donde se produce y reproduce el mundo de la vida.

Por otro lado, si se enfoca en la estructura de la RS, prevalece la perspectiva cognoscitiva y del uso del lenguaje. Y es justamente retomando la noción de representación colectiva de Durkheim (1893/1987), que Serge Moscovici desarrolla la noción de RS. En tanto hecho social, se ubica en relación de exterioridad al sujeto, imponiéndose coercitivamente como estructura determinante de la acción manifestándose a través de símbolos emblemáticos, signos e imágenes capaces de ser exteriorizados a través del lenguaje, que dan lugar a la formación de arquetipos desde los cuales se expresa la visión del mundo y se justifican las acciones. De este modo, la RS refiere a una modalidad particular de conocimiento orientado por el deber ser, lo cual posibilita prever ciertos modos de comportamiento. Desde esta perspectiva las representaciones sociales son sistemas sociocognitivos organizados a partir de un núcleo central o estructurante (Abric, 1976/2001), y constituidos por esquemas periféricos (Flament, 1987), elementos no centrales cuya determinación está más individualizada y contextualizada.

Articulando ambas perspectivas y tradiciones, se puede afirmar que el proceso de construcción de la RS tiene, por un lado, una base objetiva en función de las condiciones materiales de existencia y el imaginario colectivo, que delimita y condiciona el deber ser; a la vez que, como construcción social de la realidad, es producto de la interacción simbólicamente mediada. En tanto proceso cognitivo de un sujeto contextualizado, se lleva adelante — siguiendo a Piaget-mediante la asimilación que implica la interiorización o internalización de contenidos ya conocidos, junto a la acomodación necesaria que permite la modificación de la estructura cognitiva o del esquema comportamental para poder adaptarse y dar respuesta a lo nuevo. Estamos ante la articulación de la historia, lo dado, lo heredado, con la historicidad, lo novedoso e instituyente.

Ahora bien, siendo las RS el dato a construir, el Grupo de Discusión se propone especialmente apto para el abordaje de las mismas desde la perspectiva de Moscovici, de origen durkhemiano; mientras que el Grupo Focal es más apto como herramienta para captar el sentido mentado de la acción, en sentido weberiano (Canales, 2006). El Grupo de Discusión permite acceder a la dimensión simbólica, al significado comunitario de las categorías, de los temas sociales y culturales. La dinámica grupal permite que se despliegue el sentido y la normatividad contenida:

Hay un sentido moral como autoridad del grupo sobre el sujeto. El deber, y su contracara los ideales, refieren a las formas de representación de la comunidad, lo que Durkheim entiende como modos de hacer, pensar y sentir externos y coercitivos (...) modelos distinguidos y marcados por los sujetos como lo bueno, lo correcto o moral (Canales, 2006, p. 266). 
El análisis de las RS que deviene de la tradición durkhemiana se ubica más próxima a un uso textualista, y suele utilizar el análisis del discurso, cuyos antecedentes se encuentran en la semántica estructural, incorporando el contexto de enunciación. Mientras quienes retoman la fenomenología y la teoría de la interacción weberiana, suelen partir de una lectura transversal, desde la cual generan y definen las categorías conceptuales, lo cual “implica pasar del texto y sentido literal a categorías y relaciones subyacentes entre categorías que producen sentido en contextos específicos" (Martinic, 2006, p. 301).
En la medida en que se imbrican lo individual y lo colectivo, es posible aprehender la RS, en tanto sentido instituido, entendido como deber e ideal social que reproduce modelos de pensamiento, lenguaje y acción.

Por su lado, los Grupos Focales, permiten detectar tipificaciones e informar sobre las racionalidades que organizan la experiencia vivida, ya sea que se trate de acciones racionales con arreglo a fines, con arreglo a valores, o una combinación de ambas. Al esclarecer el sentido práctico de los mundos sociales que sostienen los consensos cognitivos sobre lo real y los esquemas de actuación, se explicita el saber o principio de realidad, o sea, el conjunto de distinciones y valoraciones desde los cuales el sujeto se posiciona, haciendo posible comprender e interpretar las RS.

\section{El análisis de las representaciones sociales}

Las vertientes teóricas a las que se hizo referencia, tienden también a delimitar el tipo de análisis que se lleve adelante.

El análisis de las RS que deviene de la tradición durkhemiana se ubica más próxima a un uso textualista, y suele utilizar el análisis del discurso, cuyos antecedentes se encuentran en la semántica estructural, incorporando el contexto de enunciación. Mientras quienes retoman la fenomenología y la teoría de la interacción weberiana, suelen partir de una lectura transversal, desde la cual generan y definen las categorías conceptuales, lo cual "implica pasar del texto y sentido literal a categorías y relaciones subyacentes entre categorías que producen sentido en contextos específicos" (Martinic, 2006, p. 301).

En términos generales, el análisis estructural comienza identificando las unidades básicas de sentido, así como también las relaciones existentes entre ellas; luego, en un segundo momento organiza dichas relaciones con base en el modelo de acción que orienta las prácticas (Martinic, 2006). En este tipo de abordaje el código constituye la unidad mínima, operando de modo relacional a través de principios, entre los que se encuentran el de disyunción, el de conjunción, el de similitud y el de contraste. Dichas enunciaciones no son neutras, sino que se enmarcan en una ética normativa que delimita la connotación positiva o negativa, la aceptación o el rechazo, tanto implícitos - que deben ser indicados entre paréntesis para indicar el carácter hipotético-. como explícitos - que se manifiestan a través de adjetivos-, generando un índice de valoración. Dicho índice se relaciona con la dimensión ética y afectiva a la que hacen referencia Durkhiem (1893/1987) y Moscovici (1961/1986), respectivamente.

En la medida en que "el análisis estructural no se dirige a comprender los efectos del discurso sobre el sujeto en particular sino sobre la estructura simbólica de la cual participa" (Martinic, 2006, p. 316), se suele trabajar con múltiples y extensos textos, por lo cual es necesario proceder reduciendo la cantidad de códigos, a través del procedimiento que permite asociarlos a una misma categoría que los incluye. El texto puede dar lugar a estructuras paralelas (principio de asociación y equivalencia de los términos); jerarquizadas (términos subordinados clasificados en cascada en función de un sistema de clasificación jerárquico); o cruzadas (complejidad y los matices combinado valoraciones positivas y negativas, sentidos opuestos simultáneamente), que dan cuenta de los principios simbólicos que subyacen al texto. Al evocar, sintetizar, o condensar un mismo significado, es posible pasar de una pluralidad de expresiones a un único término por asociación semántica, lo cual posibilita visualizar la estructura del texto. De este modo en el primer nivel descriptivo el texto da lugar a una cantidad de códigos de base que es necesario reducir, haciendo uso de 
la noción de saturación, de forma tal que se hace innecesario considerar los códigos que respondan a un mismo principio organizador, ya que uno de los supuestos del método es que la estructura está presente en el fragmento.

Por su parte, desde la perspectiva de RS en tanto interacción intersubjetiva simbólicamente mediada, el acento se coloca en los tópicos emergentes y subyacentes en los textos, que analíticamente responden a los mismos principios orientadores de sentido. Para ello se lleva a cabo un esquema organizativo de los datos en un proceso abductivo por inferencia, a partir del cual se genera teoría sustantiva, explicativa y predictiva por plausibilidad. Bajo esta modalidad de análisis del discurso se privilegia la densidad conceptual y la explicación sobre la descripción y la estructura. Desde la Grounded Theory (Glaser \&. Strauss, 1967), el procedimiento inductivo significa que la teoría esta enraizada, grounded, o fundada en el material empírico, y que la teorización o generating theory, se produce a partir de la articulación entre conceptos y materiales empíricos, en la que el trabajo de terreno corresponde a un proceso generativo y no verificatorio.

El proceso de codificación consiste en generar una denominación común al conjunto de datos que comparten un atributo, iniciando el proceso de interpretación y de relación entre la teoría y el campo. El criterio de parsimonia permite maximizar la explicación con un mínimo de casos, identificando asimismo diferencias y contradicciones; mientras el criterio de alcance permite ampliar el alcance desligándose del caso y permitiendo explicar al remitir a una teoría general o sustantiva. Comenzando, entonces, por el procedimiento analítico de codificación abierta, que identifica y agrupa categorías que fueron conceptualmente detectadas como significativas en el texto; se continúa el proceso sistematizando la codificación abierta hasta su saturación —codificación axial-; llegando así a la codificación selectiva, entendida como proceso de reducción y de generación de categorías centrales sustantivas - dando cuenta de un campo particular del universo de análisis- y categorías centrales formales -en tanto categorías transversales cuya significación teórica es abarcativa de diversos contextos y ámbitos. El pasaje de una categoría sustantiva a una categoría formal supone el descubrimiento de homologías entre procesos diferentes donde se pone en juego la misma categoría. A partir de allí es posible producir teoría, plantear hipótesis extensivas a otras situaciones y casos. La confección de mapas conceptuales facilita la visualización de las semejanzas, diferencias y contradicciones entre las RS que emergen de la escucha, el análisis y la interpretación de lo producido en el diálogo grupal (Canales, 2014).

\section{Consideraciones finales}

Retomar las tradiciones que dieron origen a las respectivas conceptualizaciones de la RS, que a su vez, derivan en diferentes herramientas metodológicas y de análisis, pretende visibilizar y profundizar en el estudio de ésta como categoría teórica y analítica que ha venido ganando terreno en la investigación social, y de este modo de evitar la reproducción de miradas dicotómicas o reduccionistas.

La relevancia y la riqueza de dicha categoría conllevan a la necesidad de combinar ambas perspectivas teóricas que, de manera complementaria, se articulen y asuman diferentes abordajes metodológicos y estrategias de análisis, sin que esto signifique un eclecticismo teórico-metodológico.

Para que sea posible visibilizar el mundo de la vida naturalizado, es necesario hacerlo explícito, tipificarlo, y significarlo. Y para formular teoría partiendo de la vivencia inter- 
subjetiva, es necesario llevar adelante un proceso analítico de segundo orden, mediante el que se pueda interpretar cómo las RS contextualizadas en el tiempo y el espacio llegan a estandarizarse, conformando las costumbres y los hábitos que son producidos y reproducidos por los actores en toda interacción simbólicamente mediada.

La vigencia de la noción de RS es particularmente relevante para una perspectiva epistemológica que concibe el proceso de investigación en el marco de una relación sujeto-sujeto, es decir, de intercambio e interacción entre sujetos, ambos sujetos-en-proceso, que se van transformando al resignificar la realidad a través de la reflexividad inherente a toda actividad conversacional, porque el sujeto "está sujetado por el orden simbólico" (lbáñez, 1991, p. 28).

Es en este sentido que, -en lo que lbáñez (1991) denomina investigación de segundo orden-, desde una sociología de la vida cotidiana, que recupera al sujeto, es ineludible enfatizar en los mecanismos transferenciales y contratransferenciales que se ponen en juego en el entretejido de los textos, en los contenidos psicosociales que van más allá de lo lingüístico en el habla, y que permiten aproximarnos a la comprensión del sentido común, para poder dar cuenta del grado y del proceso de estructuración de las representaciones sociales en un tiempo y un espacio determinado.

Una cuidadosa, abierta y atenta escucha de los universos semánticos, de los elementos cognitivos, simbólicos y subjetivos, del orden de sentido común atribuido en tanto código cultural interiorizado, permite un distanciamiento crítico de la obviedad con la que se presenta la vida cotidiana, adquiriendo así el status ontológico de realidad y de verdad. Desentrañar el proceso de construcción social de la RS, desnaturalizar aquello que ha sido instituido, permite comprender y potenciar procesos de transformación social.

Profundizar en el estudio de las RS en tanto categoría teórica y estrategia metodológica, tal como es concebida actualmente, permite el pasaje de una visión finalista y abstracta hacia la comprensión de la vida cotidiana y el sentido común; al condensar en forma analítica, sintética y denotativa, la RS permite acceder a las categorías de entendimiento de los actores, hace posible escribir y comprender los sentidos que los sujetos le dan a la acción, en tanto intérpretes y constructores, que de modo relacional van articulando el proceso de socialización adquirido, con la coyuntura presente y con las expectativas de futuro.

Queda planteado el desafío de llevar a cabo un mapeo que contemple las diversas RS que coexisten en la fragmentación social que caracteriza a las sociedades hipermodernas del Siglo XXI. La dimensión espacio-temporal articula con las interacciones simbólicamente mediadas, a la vez que la relación entre las condiciones materiales de existencia con los procesos de interiorización y apropiación, delimitan las características y particularidades que asumen las RS en la actualidad.

\section{Referencias}

Abric, J. (2001). Prácticas sociales y representaciones. http://datateca.unad.edu.co/ contenidos/403019/Entorno de Conocimiento/Lecturas Complementarias/ Abric JC Practicas Sociales y Representaciones.pdf

Acosta, M, Quiñones, M., \& Supervielle, M. (2015). Introducción a la Sociología Cualitativa. Montevideo: Comisión Sectorial de Educación Permanente. Universidad de la República. http://www.eduper.edu.uy/publicacion generica/introduccion-a-la-sociologia/ 
Araújo, A. (2013). Todos los tiempos, el tiempo. Trabajo, vida cotidiana e Hipermodernidad. Montevideo: Psicolibros. http://www.entrelibros.com.uy/Psicolibros/ Generos/Ensayo/Todos-los-tiempos-el-tiempo/flypage.tpl.html

Aristóteles. (1994). Tratados de lógica. Obra completa. Volumen I Organon. Madrid: Gredos. https://enblancoe.files.wordpress.com/2013/11/aristoteles-tratados-de-logica.pdf

Aubert, N. (2004). L'individu hypermoderne. Ramonville Saint-Agne: Èrès. https:// www.cairn.info/l-individu-hypermoderne--9782749203126.htm

Bauman, Z. (2005). Identidad. Buenos Aires: Losada. https://www.casadellibro.com/ libro-identidad/9789500393997/1090459

Bauman, Z. (2000). Modernidad líquida. Buenos Aires: Fondo de Cultura Económica. https://catedraepistemologia.files.wordpress.com/2009/05/modernidad-liquida.pdf

Berger, P., \& Luckmann, T. (1986). La construcción social de la realidad. Buenos Aires: Amorrortu. http://cmap.javeriana.edu.co/servlet/SBReadResourceServlet?rid=1 K7H81GYJ-G94DMX-2R1

Blumer, H. (1982). El Interaccionismo simbólico, perspectiva y método. Barcelona: Hora D.L. https://es.scribd.com/document/288882386/El-Interaccionismo-Simbolico-Perspectiva-y-Metodo-Blumer

Canales, M. (coord.). (2014). La escucha de la escucha. Análisis e interpretación de la investigación cualitativa. Chile: LOM Ediciones. http://www.academia.edu/25248889/Escucha de la escucha An\%C3\%A1lisis_e_interpretaci\%C3\%B3n_de_la_investigaci\%C3\%B3n_cualitativa

Canales, M. (coord.). (2006). Metodologías de Investigación Social. Introducción a los oficios. Chile: LOM Ediciones. http://www.galeon.com/alpuche932/metodo1.pdf

Castoriadis, C. (1980). La institución imaginaria de la sociedad. (Vol. 1 y 2): El imaginario social y la institución. Barcelona: Tusquets. http://www.terras.edu. ar/biblioteca/16/16TUT Castoriadis Unidad 2.pdf

Coffey, A., \& Atkinson, P. (2003). Encontrar sentido a los datos cualitativos. Estrategias complementarias de investigación. Colombia: Contus. http://www.fceia.unr. edu.ar/geii/maestria/2014/DraSanjurjo/8mas/Amanda\%20Coffey,\%20Encontar\%20el\%20sentido\%20a\%20los\%20datos\%20cualitativos.pdf

Corbin, J., \& Staruss, A. (1990). Basics of Qualitative Research: Grinded Theory, procedures and techniques. Canadá: Sage Publication, https://www.amazon.com/ Basics-Qualitative-Research-Procedures-Techniques/dp/0803932510

Durkheim, E. (1987). La división del trabajo social. Buenos Aires: Ediciones LEA, http:// www.edu.mec.gub.uy/biblioteca digital/libros../D/Durkheim,\%20Emile\%20 -\%20Division\%20del\%20trabajo\%20social.pdf

Flament, C. (1989). Structure et dynamique des représentations sociales. En D. Jodelet (Ed.). Les représentations sociales. (pp. 204-219). Paris: Presses Universitaires de France, https://www.cairn.info/load pdf.php?ID ARTICLE=PUF JODEL 2003010224

Flores, R., \& Naranjo, C. (2014). Análisis de datos cualitativos: el caso de la grounded theory (teoría fundamentada) pp. 75-113. En M., Canales (coord.), La escucha de la escucha. Análisis e interpretación de la investigación cualitativa. Chile: LOM Ediciones. http://www.academia.edu/25248889/Escucha de la escucha An\%C3\%A1 lisis_e_interpretaci\%C3\%B3n_de_la_investigaci\%C3\%B3n_cualitativa

Glaser, B., \&. Strauss, A. (1967). The discovery of grounded theory: strategies for qualitative research. New York: Aldine Publishing Compan. http://www.sxf.uevora.pt/wp-content/uploads/2013/03/Glaser 1967.pdf 
Huberman A., \& Miles, M. (1991). Qualitative, Data Analysis. Canadá: Sage Publication, http://researchtalk.com/wp-content/uploads/2014/01/Miles-Huberman-Saldana-Drawing-and-Verifying-Conclusions.pdf

Ibáñez, J. (1991). El regreso del sujeto: la investigación social de segundo orden. Madrid: Siglo XXI Editores, http://www.academia.edu/4988619/EL REGRESO DEL SUJETO_La investigaci\%C3\%B3n_social_de_segundo_orden

Jodelet, D. (1986). La representación social: teoría, método y técnica. En S. Moscovici (comp.), Psicología Social II (pp. 116-125). Buenos Aires: Paidos, http://23118. psi.uba.ar/academica/carrerasdegrado/psicologia/informacion adicional/ obligatorias/036 psicologia social2/bibliografia.html

Kant, I. (2007). Crítica de la razón pura. Buenos Aires: Colihue, http://www.biblioteca. org.ar/libros/89799.pdf

Lipovetsky, G. (2006). Los tiempos hipermodernos. Barcelona: Anagrama, http://www.anagrama-ed.es/libro/argumentos/los-tiempos-hipermodernos/9788433962478/A 352

Martinic, S. (2006). El estudio de las representaciones sociales y el análisis estructural del discurso. En M., Canales (coord.), Metodologías de Investigación Social. Introducción a los oficios. Chile: LOM Ediciones, http://www.galeon.com/alpuche932/metodo1.pdf

Moscovici, S. (comp). (1986). Psicología social. Buenos Aires: Paidós, https://books. google.com.co/books/about/Psicolog\%C3\%ADa social.html?id=Pz8Hq9QSPsYC\&redir_esc=y

Navarro Carrascal, O., \& Restrepo Ochoa, D. (2013) Representaciones Sociales: perspectivas teóricas y metodológicas. CES Psicología, 6(1), i-iv. http://148.215.2.10/ articulo.oa?id=423539419001

Pierce, C. (2007). El pragmatismo. Madrid: Ediciones Encuentro. http://www.edicionesencuentro.com/libro/el-pragmatismo.html

Weber, M. (1985). Economía y Sociedad. Conceptos sociológicos fundamentales. Montevideo: Fondo de Cultura Universitaria. https://sociologia1unpsjb.files. wordpress.com/2008/03/weber-economia-y-sociedad.pdf

Weisz, C. (2014). Imaginario, representación social y construcción identitaria en la Cooperativas Sociales. En XV Jornadas Psicología de las Organizaciones y el Trabajo. Montevideo: Facultad de Psicología, Universidad de la República. http:// www.psico.edu.uy/sites/default/files/3er. convocatoria xv jornadas pot 0.pdf 